\title{
Risk stratification by quantitation of LV dyssynchrony: A new branch of the field of nuclear cardiology
}

\author{
Marc Ovadia, $M D,{ }^{a, b}$ and Islam Abudayyeh, $M^{c}$ \\ a Electrophysiology Section, Advocate Lutheran General Hospital, Advocate Lutheran General \\ Children's Hospital, Park Ridge, IL \\ b Department of Medicine, Rosalind Franklin University of Medicine and Science, North Chicago, \\ IL \\ c Division of Cardiology, Loma Linda University Health Care, Loma Linda, CA
}

Received Mar 26, 2014; accepted Mar 26, 2014

doi: $10.1007 / \mathrm{s} 12350-014-9907-3$

\section{See related article, pp. 739-746}

\section{1}

Of the many accepted predictors of survival in an adult population, one is a measure of cardiac performance: ventricular ejection fraction.

The effort to deepen the understanding of the predictors of death in different forms of cardiac disease or in non-cardiac diseases wherein cardiac death rate is increased, has led, of course, to deeper probing of underlying non-cardiac pathophysiology, e.g., of diabetes, as well as to correlations of survival with an observable for which there may have been no recognizable direct mechanistic significance at the time of the recognition of that observable's significance.

The contribution of Aggarwal et $\mathrm{al}^{1}$ brings to the discussion a new and highly unexpected data point. In end-stage renal disease, an important and epidemic disease where cardiac death, in the sum of many forms, takes a high toll, the authors study a nuclear cardiologic measure of ventricular performance, phase bandwidth, and surprisingly they find a significant correlation with survival in the largest series studied to date (close to a thousand patients). Adding to the significance of their

Reprint requests: Marc Ovadia, MD, Electrophysiology Section, Advocate Lutheran General Hospital, Advocate Lutheran General Childrenós Hospital, 1775 Dempster Street, Park Ridge, IL 60068; movadia@uic.edu

J Nucl Cardiol 2014;21:747-52.

$1071-3581 / \$ 34.00$

Copyright (C) 2014 American Society of Nuclear Cardiology. findings, is the peculiar simplicity of the analysis, for they apply simple cut-offs to the FFT-derived first harmonic's bandwidth, and discover a clear survival advantage of smaller bandwidth. (This appears particularly important in a field of imaging where the last word has not yet been written on data analysis and optimal technique for deriving meaningful bandwidth measures, a matter to which we shall return in Section 3.)

To appreciate fully the significance of the singling out of phase bandwidth-a measure of ventricular performance quite independent of ventricular function-it is useful to start with a discussion of heart physiology.

Classical determinants of cardiac performance include contractile state (contractility, or elastance in the framework of Sagawa and co-workers ${ }^{2}$ ), ventricular myocardial mass, rate, afterload, preload and other optimizable atrial determinants of cardiac filling, and diastolic function as a ventricular determinant of ventricular filling. Contractility and optimized preload and afterload are all directly reflected in ventricular ejection fraction.

To the systolic measures, we must add synchrony explicitly. For, independently of contractile state, enhanced synchrony improves stroke volume. Consider the patient with good contractility and a large compliant apical aneurysm, a portion of stroke work will be expended in filling the aneurysm, a useless cycle that reduces cardiac output. And in other patients with dilated heart and depressed contractility, more subtle inefficiencies are introduced by dyssynchrony that similarly reduce cardiac output, other things being equal.

The pressure-volume relation that is the foundation of Braunwaldian cardiac performance analysis, does not directly reflect the presence or absence of synchronous contraction within the ventricle. In the patient with a 
large apical aneurysm, obviously, the aneurysm's volume is counted simply as part of the ventricular volume, and the wasted work of filling the aneurysm-and the emptying of the aneurysm out of phase with the ventricular emptying - are completely invisible to the analysis. Only a larger volume and reduced aortic ejection come into the equations. Now it is not due to some form of carelessness that this is overlooked in pressure-volume relation analysis, but simply to a choice of mechanistic model that excludes it specifically. In the foundational analytical work of Suga and Sagawa, for example, all models presented share the assumption, " B.3: [That a]ll ... fibers contract simultaneously.,",

The advances in physiology that underlie the appreciation of synchrony have been long in coming and may of course eventually be incorporated into mainstream models.

All this would remain an insignificant footnote in a Physiology text or obscure journal article of interest only to cardiac physiologists, but for the contribution of this paper. For this paper's data can be marshaled to make a case that synchrony (or maximized or relative synchrony) is an exceptionally important measure. Based on the data of Aggarwal et al, ${ }^{1}$ synchrony stands alone among the determinants of cardiac performance in being an independent predictor of survival. And further, it is alone in being a predictor whose utility in predicting survival may persist even in the presence of normal ejection fraction.

\section{2}

In this section, we shall review the findings of this new seminal paper in the area of risk stratification and LV function.

With a primary endpoint of all-cause mortality, 828 consecutive adult patients with end-stage renal disease were evaluated for predictors of survival from SPECT myocardial scintigraphy. They were identified as patients with renal disease severe enough to warrant consideration for renal transplantation-in fact, referral for renal transplantation was an inclusion criterion, and 1,687 consecutive adult patients were evaluated. The patients were consecutive, with a male:female ratio of 55:45 and with average serum creatinine concentration $7.9 \mathrm{mg} \cdot \mathrm{dL}^{-1}$. They were studied at a single center, The University of Alabama - Birmingham (Birmingham, AL, USA). Exclusion criteria included wide QRS (whether due to ventricular pacing or to left bundle branch block), absence of properly collected and complete data, and resolution of kidney disease (by transplant), which removed 859 patients, leaving 828 consecutive patients with end-stage renal.

Characteristics of the population were as follows. The average age was 53 years old. Diabetes mellitus was present in $60 \%$ of the patients, hypertension in almost all (94\%), and cerebrovascular disease in only $3 \%$.

Electrocardiogram was normal (mean QRS duration 0.081 seconds) and average ejection fraction was $55 \%$, with only $7 \%$ of patients having a morbidly depressed ejection fraction of $\mathrm{EF}<35 \%$. They were studied with stress and rest imaging with an isobutylnitrile class radiotracer.

Perfusion imaging was abnormal in $41 \%$, with reversible perfusion defects in only $3 \%$ and with a fixed defect in $4.8 \%$, the latter designation encompassing not only scar but also artifact and hibernating myocardium.

Mean LV mass was $167 \pm 1 \mathrm{~g}$, exceeding a normal mean by more than two standard deviations.

Synchrony was assessed by the technique of Fast Fourier Transform (FFT) applied to the three-dimensional SPECT data set. This was used to obtain just the phase distribution for the first harmonic. Phase distribution was used to derive two measures, phase bandwidth and phase standard deviation. No time domain measures were used and no measures derived from Fourier decomposition of higher harmonics were used.

The findings were quite striking.

Mortality was high-35\% during the period of follow-up. Mean LVEF was lower in the group who died than in the group that survived-52 $\pm 0.7 \%$ as compared with $56 \pm 0.5 \%$, but this difference of $4 \%$ is certainly expected both a priori, and based on the fact that that morbidly low EF was present in $9 \%$ of the patients who died vs $5 \%$ of the patients who survived. Of the other nuclear scintigraphy derived data, there was no difference in the pattern of perfusion defects in the patients who died vs those who survived.

But the phase bandwidth showed a significant difference for those who died vs those who survived $\left(73^{\circ} \pm 2.6^{\circ}\right.$ vs $\left.66^{\circ} \pm 1.8^{\circ}, P=.02\right)$. And "[A] KaplanMeier analysis revealed a stepwise increase in mortality with increasing [phase bandwidth] ... in tertiles.', 1 There was a $7.2 \%$ annual mortality for patients with higher phase bandwidth, compared with a 5.6\% annual mortality for patients with lower phase bandwidth $(P<.005)$. This is a $28 \%$ higher mortality-that is $(7.2 \%-5.6 \%) /(5.6 \%)$ - which is predicted specifically by the phase bandwidth.

This datum is obviously highly significant for the field. And it is more significant if it is proven, as this study tries to do, for the particularly tough model of chronic and end-stage renal disease, where ejection fraction is not low, or not exceptionally low, and where muscle mass is preserved or elevated in comparison with other clinical contexts where the cardiac death rate is similarly elevated. It may extrapolate to large populations with hypertension or to large populations with 
diabetes mellitus - the datum may prove foundational for hypothesis-building in nuclear cardiology.

These, to be brief, are the findings of this important study. It is on the basis of these findings that we regard this paper as seminal and prismatic. Looking back a few years from today, it is precisely this datum that will probably have thrust phase bandwidth into center stage, irrevocably, for risk stratification for death.

\section{3}

The present section comprises a critique of method. We shall review briefly the sorts of error one can make in analyzing synchrony. This goes to the question, "Is the basic finding of this seminal paper founded on solid science?',

We shall discuss first the artifacts of time domain measures, which are carefully avoided by Aggarwal et al. ${ }^{1}$ Then we will discuss shortcomings of the use of SPECT data for Fourier analysis.

The time domain measures used by other authors basically compare the timing of contraction of one wall vs the opposed wall. These measures are ubiquitous, but all share a common important artifact.

In contrast to Fourier analysis, a frequency-domain measure, these measures do not restrict analysis to movement with the same frequency as the heart beat. Therefore, both constant-velocity and random displacements will affect them.

Consider the effect on an assessment of the timing of onset of contraction of the near wall. If we parameterize wall movement as a Taylor series then right at the turnaround time the edge movement may have the equation

$$
x=x_{0}-C t^{2}+\cdots,
$$

where $x_{0}$ is a constant, the end-diastolic dimension, $C$ is a constant with units of $\mathrm{cm} \cdot \mathrm{second}^{-2}$ and ... refers to higher order terms. Thus, the wall coordinate was getting closer and closer to $x_{0}$ as time elapsed in the period prior to time $t=0$, which is the time the wall starts moving in the opposite direction, and the wall coordinate gets smaller and smaller as time elapses in the period after time $t=0$. For the far wall one has the equation

$$
x=C t^{2}+\cdots
$$

and the wall coordinate had gotten closer and closer to 0 as time elapsed in the period prior to time $t=0$, and then the coordinate gets larger and larger. (The coordinate of the midpoint of the ventricle in this parameterization is $x_{0} / 2$ ).

The fact that $t=0$ is the time of onset of contraction may be determined by inspection, or by equating the derivative to zero. For the near wall

$$
\frac{\mathrm{d} x}{\mathrm{~d} t}=-2 C t
$$

and this is equal to zero precisely at $t=0$. For the back wall,

$$
\frac{\mathrm{d} x}{\mathrm{~d} t}=+2 C t
$$

and this too is equal to zero precisely at $t=0$. Thus, the movement of these two opposed walls is seen to be synchronous.

But what is the effect of rectilinear heart movement or random heart movement? If the $x$-component if the heart's velocity right at the time of the onset of contraction is $v_{0}$, a constant, then the dynamical equations are

$$
x=x_{0}+v_{0} \cdot t-C t^{2}+\cdots
$$

for one wall and

$$
x=v_{0} \cdot t+C t^{2}+\cdots
$$

for the other.

Taking the derivative of each of these, we get

$$
\frac{\mathrm{d} x}{\mathrm{~d} t}=v_{0}-2 C t
$$

for the first and

$$
\frac{\mathrm{d} x}{\mathrm{~d} t}=v_{0}+2 C t
$$

for the second. When we equate each of these to zero, we find the artifact: the apparent onset of contraction has been shifted forward by

$$
\frac{v_{0}}{2 C}
$$

for the one wall, and backward by the same amount for the other, so that there appears to be dyssynchrony, when there really is not. And two opposite walls that appear synchronous by this time domain measure, actually are not synchronous, but have a difference of timing of

$$
\frac{v_{0}}{C}
$$

between them. Since it is obviously impracticable to have anything like a moving detector synchronized to the movement of the center of mass of the intraventricular blood of the heart, it is perhaps impossible to eradicate this artifact. Hence time domain measures can not easily be used in synchrony studies. As mentioned, Aggarwal et al are to be complimented for correctly avoiding any time domain measure altogether, so this artifact does not exist in the present paper. ${ }^{1}$ We agree with Aggarwal et al that all measures of synchrony or dyssynchrony should be valid frequency-domain measures.

We turn now to certain shortcomings that attend the use of SPECT data for Fourier analysis. 
Fourier phase imaging was introduced for MUGA $^{3-9}$ and is based on the use of the Cooley-Tukey FFT, applicable to MUGA and first-transit studies. For a certain class of wall movements, however, there is a significant difference between application of the methodology to SPECT imaging of isobutylnitrile class perfusion tracers, vs MUGA.

In MUGA and in first-transit radionuclide angiocardiography, obviously the blood pool is the emitter (is "lit up"). Consider the differences inhering in the use of Cooley-Tukey FFT for estimating phase relevant to the contraction of normal heart, when the wall is lit up, and the blood pool is not. Now the wall is not simply a boundary (like the edge of the blood pool in a gated blood pool [MUGA] scan), rather it is a lamina. A lamina differs from a boundary in that a lamina is constituted by two boundaries, that is, the boundary between the non-emitting lumen and the emitting wall, and then adjacent to this the boundary between the emitting wall and the extracardiac nonemitting space. Each point on the wall may be viewed as a source to which a point source function applies. ${ }^{10}$ The wall is a lamina or surface, each point of which is a point source. Now the lamina translates radially a distance on the order of $14 \mathrm{~mm}$ (in the absence of severely depressed left ventricular function), an excursion that exceeds the typical thickness of a wall. Taking the point source function to be a function of $x$ and choosing $x$ to coincide with a ventricular radius (Figure 1A-C), the effect of cardiac contraction is to translate the wall back and forth across the voxel. Let this voxel be at $x=0$. (The choice of origin is obviously arbitrary and a constant translation may be performed once for all points with no loss of generality.) We proceed to derive the time-activity curve for a certain class of wall movements relevant in this case, from the dynamical equation for a simple model, and from the standard point source function.

Now the functionality of the point source function may be assumed to be

$$
A \cdot e^{-x^{2}}
$$

which we can write as

$$
e^{-x^{2}}
$$

without loss of generality by choosing units to make the maximum of $A$ equal to $1 .{ }^{1}$

\footnotetext{
${ }^{1}$ Units of distance are discussed below and in the figure legend but the present discussion regards amplitude units. $A$ is essentially a constant. Thus, the amplitude may be normalized so that $1 \mathrm{U}$ is equal to the maximum value of the function along the radius. This factor $A$ is equal to the $L_{\infty}$ (supremum) norm
}

$$
|A(x, t)|_{\infty}=\operatorname{ess} \sup |A(x, t)|,
$$
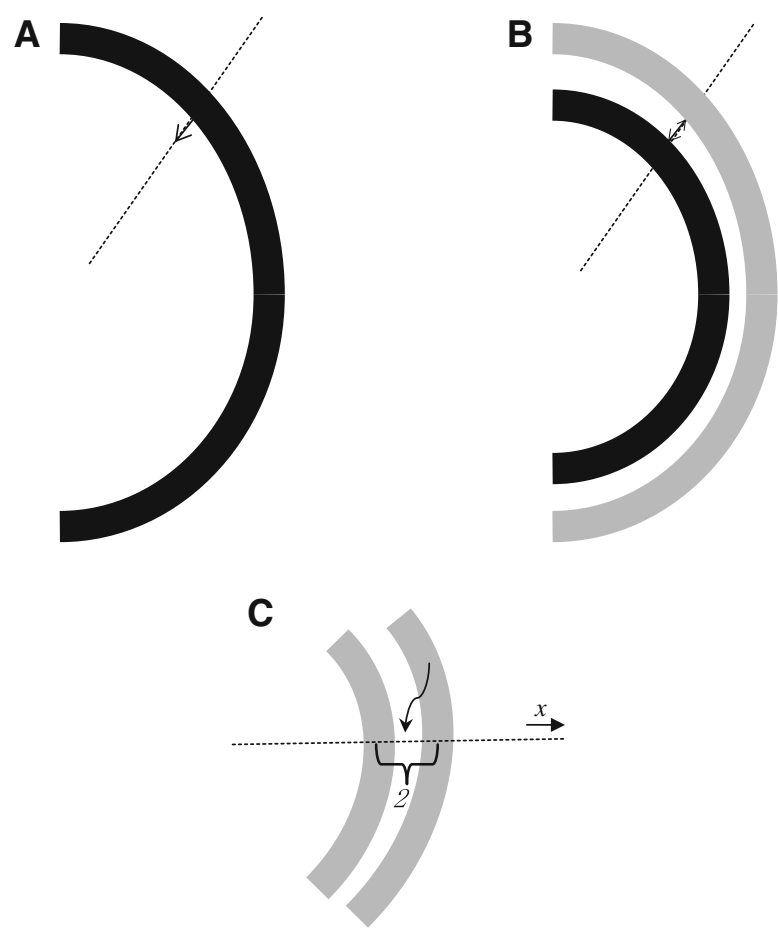

Figure 1. (A) The ventricular myocardium at end-diastole is depicted in black. The excursion is indicated by the oneheaded arrow, and the ventricular radius along which the excursion will occur is indicated by a dotted line. (B) The ventricular myocardium at end-systole is depicted now in black, and the previously drawn (A) ventricular myocardium at end-diastole is now depicted in gray. The ventricular radius along which excursion occurs sinusoidally is again indicated by the dotted line. A two-headed arrow partially identifies the excursion. (C) The previously drawn representation is redrawn, rotated to the right. The ventricular radius is used as the $x$-axis, with the Origin (indicated by the curved arrow) chosen to be in the middle of the excursion. Distance normalization is indicated by the curved bracket, where units of distance along $x$ are so chosen that the excursion is defined as $2 \mathrm{U}$. The rationale for this is that the full oscillation $\pm \sin t$ or $\pm \cos t$ equals $2 \mathrm{U}$.

The effect of translation, for the class of wall movements that we wish to consider, is

$$
x \rightarrow x-\sin \omega t,
$$

where the lowercase $\omega$ is the fundamental frequency, or the so-called first harmonic. By taking the coefficient of $\sin \omega t$ to be unity we are restricting the group of movements to those having a certain relationship to wall thickness. Also we will make the assumption, correct because of the abnormally thick heart, that apart from translation, the functionality of the source changes little during systole. (While this applies to a hypertrophied

\section{Footnote 1 continued}

which denotes the smallest value that bounds the function everywhere along the radius at any time. ${ }^{11}$ 
heart, the same assumption may be correct for different reasons in a thin or scarred wall that is displaced during cardiac contraction without appreciable thickening.)

Therefore, the correct function for a voxel of interest along this radius is

$$
e^{-(x-\sin \omega t)^{2}}
$$

and for what is of interest to us, the voxel at the midpoint of the excursion where $x \sim 0$, we have

$$
e^{-\sin ^{2} \omega t}
$$

But this may be written out

$$
1-\sin ^{2} \omega t+\frac{\sin ^{4} \omega t}{2}-\frac{\sin ^{6} \omega t}{6}+\cdots+
$$

a convergent infinite series that may be approximated by any partial sum with remainder less than the subsequent term-since this is an alternating series ${ }^{12,13}$ — which means that for an $n$ term approximation the remainder approaches zero as $1 / n$ ! or is $o(1 / n !)$ using the LandauSymbol $o .^{14}$

Choosing to approximate this by the first two terms only, we have, by a trigonometric identity

$$
1-\sin ^{2} \omega t \equiv \frac{1}{2}+\frac{1}{2} \cos 2 \omega t
$$

and this is a correct expression for the functional form of the voxel's time-activity curve for this class of wall movements at this location on the radius.

This being the functional form of the voxel's timeactivity curve, the Cooley-Tukey FFT algorithm as used here is incomplete and possibly inadequate (if not inappropriate), since there is no $\pm \sin \omega t$ and no $\pm \cos \omega t$ term whatsoever. There is simply no fundamental frequency dependency of this voxel's time-activity curve for this class of movement.

Other authors have addressed this problem, in the pages of this journal, by use of higher harmonics. The authors of the paper under discussion cite one of those studies where FFT-derived measures of synchrony in end-stage renal disease patients included a higher harmonic, that of Chen et al. ${ }^{15}$ But the authors do not question the analytical assumption that only the first harmonic phase is an important figure of merit in assessing synchrony. That previous work's data, however, refute that assumption, for the second harmonic's amplitude coefficient is of exactly the same order of magnitude as that of the first harmonic, and the derivation presented above gives reason to believe that it is no artifact or accident for this to have been the case. In that paper, the third harmonic's coefficient is a tenth as large, so by the Riesz-Fischer theorem ${ }^{16}$ and by a consideration of the sources of noise, this three-harmonic data reduction has much to commend itself, theoretically, and two harmonics could well be adequate. But one harmonic may fail to capture all the important information about synchrony-one harmonic, as is presented here, will simply fail to capture the wall movement information at a location close to the wall edge for the class of wall movements identified here.

Fitting with a FFT algorithm that goes up to higher harmonics is not the only efficient data reduction approach available. The actual functionality may allow direct fitting using a series whose terms are Hermite polynomials $^{17}$ with the argument being a trigonometric function, although the isomorphic Weber-Hermite functions (the so-called parabolic cylindrical functions ${ }^{18}$ ) could also be used. Both are orthogonal function systems, and hence introduce computational economies, albeit different in each case. But the approach of using the FFT algorithm as Chen et $\mathrm{al}^{15}$ did in their end-stage renal disease patients, and going up to one higher harmonic, may have much to commend it. A yet simpler approach, however, may be to revert to the lost art of first-transit acquisition in radionuclide angiocardiography, where much smaller mathematical challenges exist.

\section{4}

In summary, an important or seminal contribution by Aggarwal et $\mathrm{al}^{1}$ thrusts to center stage the measurement of dyssynchrony, by showing that a $28 \%$ increment in mortality may be discerned by attempting to quantitate increased left ventricular dyssynchrony. With this monumental finding for risk stratification in cardiovascular disease, a hitherto obscure literature is suddenly thrust to the fore, and one anticipates an exciting period of critique, of method development, of hypothesisbuilding, and of clinical studies in what promises to be "the dramatic growth phase of a new branch",19 of nuclear cardiology.

\section{References}

1. Aggarwal H, Al-Jaroudi WA, Mehta S, Mannon R, Heo J-Y, Iskandrian AI, et al. The prognostic value of left ventricular mechanical dyssynchrony using gated myocardial perfuasion imaging in patients with end-stage renal disease. J Nucl Cardiol 2014. doi:10.1007/s12350-014-9886-4.

2. Suga H, Sagawa K. Mathematical interrelationship between instantaneous ventricular pressure-volume ratio and myocardial force-velocity relation. Ann Biomed Eng 1972;1:160-81.

3. Pavel DG, Byrom E, Lam W, Meyer-Pavel C, Swiryn S, Pietras R. Detection and quantitation of regional wall motion abnormalities using phase analysis of equilibrium gated studies. Clin Nucl Med 1983;8:315-21.

4. Geffers H, Adams W-E, Bitter F, Sigel H, Kampmann H. Data processing and functional imaging in radionuclide ventriculography. In: Proceedings of the international conference on information processing in medical imaging. Oak Ridge, TN: 
Biological Computing Technology Information Center; 1977. p. 322-332.

5. Bossuyt A, Deconinck F, Lepoudre R, Jonckheer M. The temporal Fourier transform applied to functional isotopic imaging. Les Colloques de l'INSERM 1979;88:397.

6. Adam W-E, Tarkowska A, Bitteer F, Stauch M, Geffers H. Equilibrium (gated) radionuclide ventriculography. Cardiovasc Radiat 1979;2:161.

7. Bitter F, Adam W-E, Geffers H, Sigel H, Kampmann H. Synchronized steady-state heart investigations. In: Garsou J, Gordenne W, Merchie G, editors. Proceedings of the international symposium on fundamentals in technical progress: Ultrasound, dose planning and nuclear medicine, vol. 3. Liège: Presses universitaires de Liège; 1979. p. 9.1-9.5.

8. Adam W-E, Clausen M, Hellwig D, Henze E, Bitter F. Radionuclide ventriculography (equilibrium gated blood pool scanning) Its present clinical position and recent developments. Eur J Nucl Med Mol I 1988;13:637-47.

9. Standke R, Maul FD, Hör G. Vollautomatische ÄquilibriumRadionuklidventrikulographie: Sektorale Ejektionsfraktion und Phasenanalyse. In: Hör G, Kaltenbach M, Maul FD, Pabst HW, editors. Interventionelle Nuklearkardiologie. Frankfort am Main, FRG: Kern und Birner; 1985. p. 220-31.

10. Galt JR, Garcia EV, Robbins WL. Effects of myocardial wall thickness on SPECT quantitation. IEEE Trans Med Imaging 1990;9:144-50.
11. Robinson JC. Infinite-dimensional dynamical systems. Cambridge: Cambridge University Press; 2001. p. 28.

12. Courant R. (McShane EJ, Trans.) Differential and integral calculus, vol. 1. 2nd ed. New York: Interscience; 1937. p. 370.

13. Whittaker ET, Watson GN. A course of modern analysis. Cambridge: Cambridge University Press; 1945. p. 18.

14. Landau E. Darstellung und Begründung einiger neuerer Ergebniße der Funktionentheorie. Berlin: Julius Springer; 1916. p. 5.

15. Chen J, Kalogeropoulos AP, Verdes L, Butler J, Garcia EV. Leftventricular systolic and diastolic dyssynchrony as assessed by multiharmonic phase analysis of gated SPECT myocardial perfusion imaging in patients with end-stage renal disease and normal LVEF. J Nucl Cardiol 2011;18:299-308.

16. Courant R, Hilbert D. Methoden der mathematischen Physik, vol. 1. Berlin: Julius Springer; 1931. p. 93-4.

17. Weyl H (Robertson HP, Trans.). The theory of groups and quantum mechanics. Mineola: Dover; 1950. p. 56-59.

18. Functions of the parabolic cylinder and of the paraboloid of revolution. In: Erdélyi A, the Staff of The Bateman Manuscript Project, editors. Higher transcendental functions, Chap. VIII, vol. 2. New York: McGraw Hill; 1953. p. 115-132.

19. Montroll E, Vineyard GH, Lévy M. In: Schwartz L, editor. Application of distributions to the theory of elementary particles in quantum mechanics. New York: Gordon and Breach; 1968. p. 5. 